Acta Crystallographica Section E

Structure Reports

Online

ISSN 1600-5368

Editors: W. Clegg and D. G. Watson

\title{
2,9-Dichloroquino[2,3-b]acridine-7,14(5H,12H)-dithione dimethylformamide disolvate
}

Takatoshi Senju, Tomonori Hoki and Jin Mizuguchi

Copyright $(\mathcal{C}$ International Union of Crystallography

Author(s) of this paper may load this reprint on their own web site provided that this cover page is retained. Republication of this article or its storage in electronic databases or the like is not permitted without prior permission in writing from the IUCr. 
Acta Crystallographica Section E

Structure Reports

Online

ISSN 1600-5368

Takatoshi Senju, Tomonori Hoki and Jin Mizuguchi*

Department of Applied Physics, Graduate School of Engineering, Yokohama National University, Tokiwadai 79-5, Hodogaya-ku, Yokohama 240-8501, Japan

Correspondence e-mail: mizu-j@ynu.ac.jp

Key indicators

Single-crystal $\mathrm{X}$-ray study

$T=93 \mathrm{~K}$

Mean $\sigma(\mathrm{C}-\mathrm{C})=0.003 \AA$

$R$ factor $=0.034$

$w R$ factor $=0.096$

Data-to-parameter ratio $=12.5$

For details of how these key indicators were automatically derived from the article, see http://journals.iucr.org/e.

\section{2,9-Dichloroquino[2,3-b]acridine-7,14(5H,12H)- dithione dimethylformamide disolvate}

The title compound, $\mathrm{C}_{20} \mathrm{H}_{10} \mathrm{Cl}_{2} \mathrm{~N}_{2} \mathrm{~S}_{2} \cdot 2 \mathrm{C}_{3} \mathrm{H}_{7} \mathrm{NO}$, is a dimethylformamide (DMF) disolvate of DTQ-Cl, which is a thionated pigment derivative of 2,9-dichloroquinacridone. The DTQ-Cl molecule is centrosymmetric and entirely planar. Two DMF molecules are hydrogen-bonded to DTQ-Cl, through the $\mathrm{NH}$ group of DTQ-Cl and the $\mathrm{O}$ atom of DMF. The molecular plane of DMF is twisted with respect to the skeleton of DTQCl by $21.4(1)^{\circ}$.

\section{Comment}

The title compound, (I), is a DMF disolvate of DTQ-Cl, which is a thionated derivative of 2,9-dichloroquinacridone known as an industrially important red pigment (Herbst \& Hunger, 1997). We have previously reported that thionation of unsubstituted quinacridone brings about an intense near-IR absorption that can be utilized for laser printers, as well as optical disks based on GaAsAl laser diodes (Mizuguchi et al., 1992, 1994). DTQ-Cl has, therefore, been synthesized in the expectation that it exhibits even better performance and stability for electronic applications. Since optical absorption in the solid state depends largely on intermolecular interactions, $\mathrm{X}$-ray crystal structure analysis of DTQ-Cl is crucial. We obtained three types of solvated crystals: one was isolated from a DMF solution (present study) and the other two were from a single solution in dimethylacetamide (Senju et al., 2005; Hoki et al., 2005). This report deals with the title dimethylformamide solvate, DTQ-Cl-2DMF, (I).<smiles>CN(C)C(=O)CO[C@@H]1c2cc3c(=S)c4cc(Cl)ccc4n4c3cc2C(=S)c2cc(Cl)ccc2N14</smiles>

(I)
Received 25 April 2005 Accepted 27 April 2005 Online 14 May 2005
Fig. 1 shows an ORTEPIII (Burnett \& Johnson, 1996) plot of (I) including the two solvent molecules. The DTQ-Cl molecule is centrosymmetric and entirely planar, as characterized by the mean deviation of $0.006 \AA$ from the leastsquares plane $(\mathrm{C} 1-\mathrm{C} 10 / \mathrm{N} 1)$. 


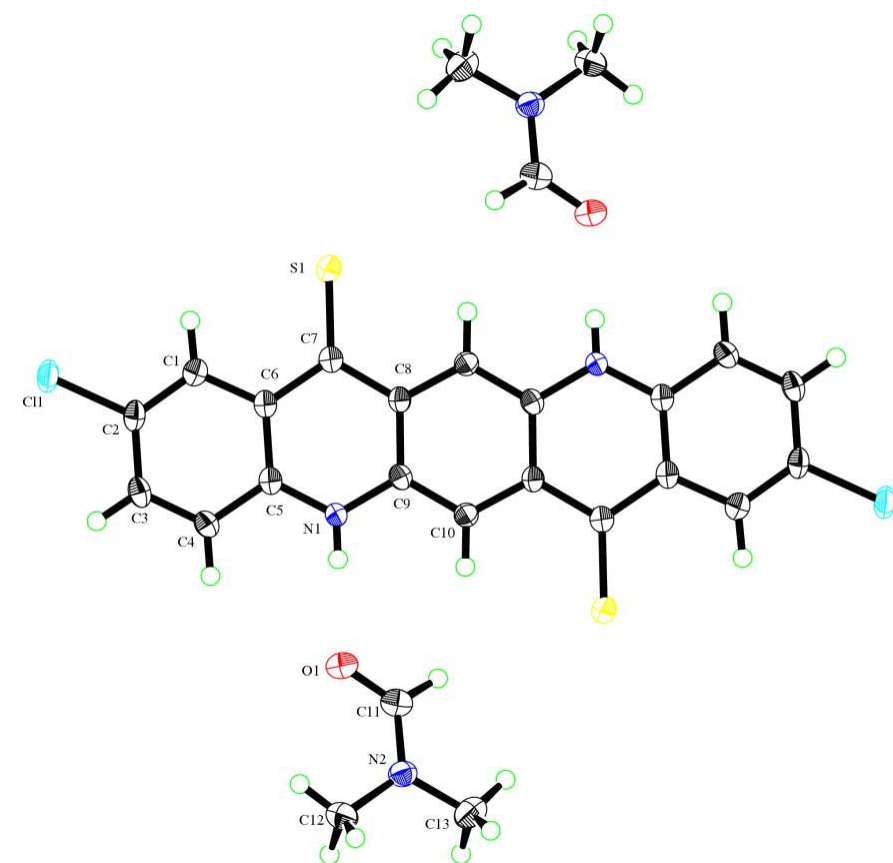

Figure 1

A view of the molecular structure of (I), showing $50 \%$ probability displacement ellipsoids. Unlabelled atoms are related to labelled atoms by the symmetry operator $(-x, 2-y,-z)$.

There are $\mathrm{N}-\mathrm{H} \cdots \mathrm{O}$ intermolecular hydrogen bonds between the NH group of DTQ-Cl and the $\mathrm{O}$ atom of DMF. As shown in Table 2, the $\mathrm{N}, \mathrm{H}$ and $\mathrm{O}$ atoms are perfectly aligned, with an N...O distance of 2.769 (2) $\AA$, indicating that this hydrogen bond is quite strong. The molecular plane of DMF is twisted with respect to the skeleton of DTQ-Cl by $21.4(1)^{\circ}$. DTQ-Cl molecules are stacked along the $b$ axis in a herringbone fashion.

\section{Experimental}

DTQ-Cl was synthesized by thionation of commercially available 2,9dichloroquinacridone, using Lawesson's reagent (Rochat et al., 1988). Crystals of (I) were grown by gradual cooling of a dimethylformamide solution prepared at about $420 \mathrm{~K}$.

\section{Crystal data}

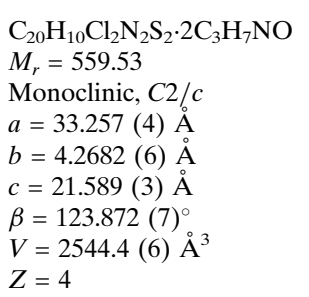

$D_{x}=1.461 \mathrm{Mg} \mathrm{m}^{-3}$

$\mathrm{Cu} K \alpha$ radiation

Cell parameters from 9508 reflections

$\theta=3.2-68.3^{\circ}$

$\mu=4.10 \mathrm{~mm}^{-1}$

$T=93.1 \mathrm{~K}$

Needle, dark green

$0.50 \times 0.10 \times 0.05 \mathrm{~mm}$

\section{Data collection}

$\begin{array}{ll}\text { Rigaku R-AXIS RAPID-F imaging } & 2123 \text { independent reflections } \\ \text { plate diffractometer } & 1947 \text { reflections with } F^{2}>2 \sigma\left(F^{2}\right) \\ \omega \text { scans } & R_{\text {int }}=0.028 \\ \text { Absorption correction: multi-scan } & \theta_{\max }=68.2^{\circ} \\ \quad(A B S C O R ; \text { Higashi, } 1995) & h=-40 \rightarrow 40 \\ T_{\min }=0.280, T_{\max }=0.815 & k=-4 \rightarrow 4 \\ 11564 \text { measured reflections } & l=-24 \rightarrow 25\end{array}$

\section{Refinement}

Refinement on $F^{2}$

$R\left[F^{2}>2 \sigma\left(F^{2}\right)\right]=0.034$

$w R\left(F^{2}\right)=0.096$

$S=1.10$

2123 reflections

170 parameters

$\mathrm{H}$ atoms treated by a mixture of

independent and constrained

refinement

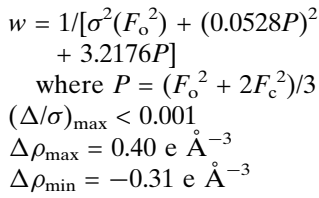

Table 1

Selected geometric parameters $\left(\AA,^{\circ}\right)$.

\begin{tabular}{llll}
\hline $\mathrm{C} 1-\mathrm{C} 2$ & $1.746(2)$ & $\mathrm{C} 4-\mathrm{C} 5$ & $1.418(3)$ \\
$\mathrm{S} 1-\mathrm{C} 7$ & $1.663(3)$ & $\mathrm{C} 5-\mathrm{C} 6$ & $1.411(3)$ \\
$\mathrm{N} 1-\mathrm{C} 5$ & $1.357(2)$ & $\mathrm{C} 9-\mathrm{C} 10$ & $1.393(2)$ \\
$\mathrm{N} 1-\mathrm{C} 9$ & $1.372(2)$ & $\mathrm{C} 8-\mathrm{C} 9$ & $1.418(3)$ \\
$\mathrm{C} 1-\mathrm{C} 2$ & $1.371(3)$ & $\mathrm{C} 8-\mathrm{C} 7$ & $1.462(2)$ \\
$\mathrm{C} 1-\mathrm{C} 6$ & $1.413(2)$ & $\mathrm{C} 8-\mathrm{C} 10^{\mathrm{i}}$ & $1.394(3)$ \\
$\mathrm{C} 2-\mathrm{C} 3$ & $1.404(4)$ & $\mathrm{C} 7-\mathrm{C} 8$ & $1.462(2)$ \\
$\mathrm{C} 3-\mathrm{C} 4$ & $1.364(3)$ & $\mathrm{C} 7-\mathrm{C} 6$ & $1.458(3)$ \\
& & & \\
$\mathrm{Cl} 1-\mathrm{C} 2-\mathrm{C} 1$ & $119.9(2)$ & $\mathrm{C} 1-\mathrm{C} 6-\mathrm{C} 7$ & $121.4(2)$ \\
$\mathrm{Cl} 1-\mathrm{C} 2-\mathrm{C} 3$ & $118.4(1)$ & $\mathrm{C} 1-\mathrm{C} 6-\mathrm{C} 5$ & $118.2(2)$ \\
$\mathrm{S} 1-\mathrm{C} 7-\mathrm{C} 8$ & $121.7(2)$ & $\mathrm{C} 2-\mathrm{C} 3-\mathrm{C} 4$ & $119.4(2)$ \\
$\mathrm{S} 1-\mathrm{C} 7-\mathrm{C} 6$ & $122.9(1)$ & $\mathrm{C} 3-\mathrm{C} 4-\mathrm{C} 5$ & $120.2(2)$ \\
$\mathrm{N} 1-\mathrm{C} 5-\mathrm{C} 6$ & $121.2(2)$ & $\mathrm{C} 4-\mathrm{C} 5-\mathrm{C} 6$ & $120.3(2)$ \\
$\mathrm{N} 1-\mathrm{C} 5-\mathrm{C} 4$ & $118.5(2)$ & $\mathrm{C} 5-\mathrm{C} 6-\mathrm{C} 7$ & $120.4(2)$ \\
$\mathrm{C} 9-\mathrm{N} 1-\mathrm{C} 5$ & $122.2(2)$ & $\mathrm{C} 9-\mathrm{C} 8-\mathrm{C} 7$ & $121.0(2)$ \\
$\mathrm{N} 1-\mathrm{C} 9-\mathrm{C} 8$ & $119.8(2)$ & $\mathrm{C} 10-\mathrm{C} 9-\mathrm{C} 8$ & $120.8(2)$ \\
$\mathrm{N} 1-\mathrm{C} 9-\mathrm{C} 10$ & $119.4(2)$ & $\mathrm{C} 9-\mathrm{C} 10-\mathrm{C} 8{ }^{\mathrm{i}}$ & $121.6(2)$ \\
$\mathrm{C} 1-\mathrm{C} 2-\mathrm{C} 3$ & $121.7(2)$ & $\mathrm{C} 8-\mathrm{C} 7-\mathrm{C} 6$ & $115.4(2)$ \\
$\mathrm{C} 6-\mathrm{C} 1-\mathrm{C} 2$ & $120.1(2)$ & $\mathrm{C} 10-\mathrm{C} 8-\mathrm{C} 7$ & $121.4(2)$ \\
\hline
\end{tabular}

Symmetry code: (i) $-x, 2-y,-z$.

Table 2

Hydrogen-bond geometry $\left(\AA{ }^{\circ}\right)$.

\begin{tabular}{lllll}
\hline$D-\mathrm{H} \cdots A$ & $D-\mathrm{H}$ & $\mathrm{H} \cdots A$ & $D \cdots A$ & $D-\mathrm{H} \cdots A$ \\
\hline $\mathrm{N} 1-\mathrm{H} 1 N \cdots \mathrm{O} 1$ & $0.82(2)$ & $1.95(3)$ & $2.769(2)$ & $180(3)$ \\
\hline
\end{tabular}

The position of the imide $\mathrm{H}$ atom was found in a difference Fourier map and refined freely along with an isotropic displacement parameter. Methyl $\mathrm{H}$ atoms were constrained to an ideal geometry, with $\mathrm{C}-\mathrm{H}=0.98 \AA$ and $U_{\text {iso }}(\mathrm{H})=1.2 U_{\text {eq }}(\mathrm{C})$, but each group was allowed to rotate freely about its $\mathrm{C}-\mathrm{C}$ bond. All other $\mathrm{H}$ atoms were placed in geometrically idealized positions and constrained to ride on their parent atoms, with $\mathrm{C}-\mathrm{H}=0.95 \AA$ and $U_{\text {iso }}(\mathrm{H})=1.2 U_{\text {eq }}(\mathrm{C})$.

Data collection: PROCESS-AUTO (Rigaku, 1998); cell refinement: PROCESS-AUTO; data reduction: CrystalStructure (Rigaku/ MSC and Rigaku, 2004); program(s) used to solve structure: SHELXS97 (Sheldrick, 1997); program(s) used to refine structure: SHELXL97 (Sheldrick, 1997); molecular graphics: ORTEPIII (Burnett \& Johnson, 1996); software used to prepare material for publication: CrystalStructure.

\section{References}

Burnett, M. N. \& Johnson, C. K. (1996). ORTEPIII. Report ORNL-6895. Oak Ridge National Laboratory, Tennessee, USA.

Herbst, W. \& Hunger, K. (1997). Industrial Organic Pigments, 2nd ed., pp. 454474. Weinheim: VCH. 


\section{organic papers}

Higashi, T. (1995). ABSCOR. Rigaku Corporation, Tokyo, Japan.

Hoki, T., Senju, T. \& Mizuguchi, J. (2005). Acta Cryst. E61. Submitted.

Mizuguchi, J. \& Rihs, G. (1992). Acta Cryst. C48, 1553-1555.

Mizuguchi, J., Rochat, A. C. \& Rihs, G. (1994). Ber. Bunsenges. Phys. Chem.

98, 19-28.

Rigaku (1998). PROCESS-AUTO. Rigaku Corporation, Tokyo, Japan.
Rigaku/MSC and Rigaku (2004). CrystalStructure. Version 3.6.0. Rigaku/MSC, 9009 New Trails Drive, The Woodlands, TX 77381-5209, USA, and Rigaku Corporation, Akishima, Tokyo, Japan.

Rochat, A. C., Jaffe, E. E. \& Mizuguchi, J. (1988). US Patent No. 4760004. Senju, T. Hoki, T. \& Mizuguchi, J. (2005). Acta Cryst. E61. Submitted. Sheldrick, G. M. (1997). SHELXS97 and SHELXL97. University of Göttingen, Germany. 